\title{
How Many Endangered Species Remain to Be Discovered in Brazil?
}

\author{
Stuart L. Pimm ${ }^{1, \star}$, Clinton N. Jenkins ${ }^{2}$, Lucas N. Joppa ${ }^{3}$, David L. Roberts ${ }^{4,5}$ \& Gareth J. Russell ${ }^{6,7}$
}

${ }^{1}$ Nicholas School of the Environment, Duke University, PO Box 90328, Durham, NC 27708, USA

${ }^{2}$ Department of Biology, 1210 Biology-Psychology Building, University of Maryland, College Park, MD 20742, USA

${ }^{3}$ Microsoft Research, $7 \mathrm{~J} J$ Thomson Avenue, Cambridge, CB3 OFB, UK

${ }^{4}$ Durrell Institute of Conservation and Ecology, School of Anthropology and Conservation, University of Kent, Marlowe Building, Canterbury, Kent, CT2 7NR, UK

${ }^{5}$ Royal Botanic Gardens, Kew, Richmond, Surrey, TW9 3AB, UK

${ }^{6}$ Department of Biological Sciences, New Jersey Institute of Technology, Newark, NJ 07102, USA

${ }^{7}$ Department of Biological Sciences, Rutgers University, Newark, NJ 07102, USA

\begin{abstract}
How many species are likely as-yet unknown to science? Even in relatively well-known groups, there may be substantial numbers of such species. It seems likely that these unknown species will be rare and threatened with extinction. Indeed, science may not discover them before they go extinct. We address these issues for a sample of endemic flowering plants and three vertebrate groups: amphibians, birds, and mammals, all from Brazil. We predict the likely numbers of missing species from models of the declining numbers of species described per five-year interval. The raw numbers increase over time, so we must scale these by the taxonomic effort. We show that while the catalogues of birds and mammals are nearly complete, the numbers of amphibians may increase by $15 \%$ and the numbers of endemic plants by $\sim 10$ to $\sim 50 \%$ depending on region. These percentages may still seem encouragingly low, given the complexities of studying a country as large as Brazil, with its extraordinary diversity, and with many of its regions large and still poorly explored. What is more worrying is that these numbers of as-yet unknown species are broadly the same as the percentages of species that are presently considered threatened with extinction. That is, we know only half of the species in danger of extinction - and our knowledge of even those species has mostly been acquired in the last three decades.
\end{abstract}

Key words: Brazil, Biodiversity, Unknown Species, Endangered Species, Amphibians, Birds, Mammals, Flowering Plants

\section{Introduction}

Brazil has extraordinary biodiversity with a greater number of terrestrial species of well-known taxa than most other countries in the world. It is likely to be comparably richer in the taxa that are poorly known, too. The range of habitats, which include the moist forests of the Amazon and the Mata Atlântica, as well as drier habitats and wetlands, surely guarantee that. Brazil also has large numbers of endangered species too, a consequence of extensive habitat destruction. These factors, combined with the remoteness of much of the country, beg our asking: how many species are likely as-yet unknown to science? Even in relatively well-known groups, there may be substantial numbers of such species.

\footnotetext{
*Send correspondence to: Stuart L. Pimm

Nicholas School of the Environment, Duke University,

PO Box 90328, Durham, NC 27708, USA

E-mail: stuartpimm@me.com
}

It seems likely that these unknown species will be rare and threatened with extinction. If they were not rare, then scientists would likely already have found and described them. Indeed, science may not discover them before they go extinct. These questions seem especially pressing in 2010, the International Year of Biodiversity, and given the commitments countries have made to document and protect biodiversity in the Convention on Biodiversity.

We address these issues for a sample of flowering plants and three vertebrate groups: amphibians, birds, and mammals. Using methods developed elsewhere (Joppa et al. 2010), we predict the likely numbers of missing species. We show that while the catalogues of all Brazil's birds and mammals are likely nearly complete, the numbers of Brazil's amphibians may increase by $15 \%$ and the numbers of just endemic flowering plants by $\sim 10$ to $\sim 50 \%$. 
For the three vertebrate groups we use estimates of the size of the geographical ranges to show that there is a strong tendency for species with large ranges to be discovered early. Recent discoveries are almost always of species with small ranges. The numbers of species with ranges $<20,000 \mathrm{~km}^{2}$ have increased rapidly in recent decades -especially for amphibians. That threshold, combined with evidence of continuing habitat destruction, constitutes grounds for the IUCN to declare a species threatened with extinction. This means that substantial numbers of as-yet unknown species will be added to the lists of endangered species. Simply, Brazil has many more threatened species than already recognized.

\section{Methods}

A surprising first question in estimating the numbers of species in a taxon is how many valid species have taxonomists already described. The problem is that taxonomists give different names to the same species inadvertently. This means we must use data sources where the sometimes-large fractions of synonyms have been removed. Following Joppa et al. (2010), we used the World Checklist of Selected Plant Families 2008 (WCSP), a unique and continuously updated synonymised world list of plants from the Royal Botanic Gardens, Kew (http://apps.kew.org/wcsp/). It has resolved the problems of synonyms for monocots, but only for some selected non-monocot families, for a total of about 110,000 species of seed plants. Second, we use GrassBase, a similar list for the roughly 10,000 species of grasses (Clayton et al. 2009).

We calculated range sizes for the amphibians, mammals, and birds from extent-of-occurrence maps projected in an equal-area projection. Bird data are from Ridgely et al. (2007), mammal data are from the Global Mammal Assessment (IUCN et al. 2008a), amphibian data are from the Global Amphibian Assessment (IUCN et al. 2008b). Only native ranges were included. The range sizes for birds are of breeding range only. Dates of species' description were from the same sources.

The second question: how should one estimate the number of species remaining to be discovered? Recent attempts employ differing methods of extrapolation of the number of species described over time, with the expectation that these numbers of new species per time interval will decline as the pool of unknown species diminishes (Solow and Smith 2005, Wilson and Costello 2005). Joppa et al. (2010) show that generally, they do not. We confirm that for most of the taxa we consider here.

Joppa et al. (2010) found these previous attempts wanting because none includes the number of taxonomists involved in describing species. The number of taxonomists active in any period has increased over the 250 years of taxonomic history. The raw numbers of species described per period has increased correspondingly. By analogy to fishing statistics, Joppa et al. (2010) scaled the raw numbers of species "caught" by taxonomists, by the "effort" required to catch them (the number of taxonomists) to produce "catch per unit effort" - the numbers of species described per unit time per taxonomist.

We restricted our plant analyses to species endemic to five regions of Brazil that the plant databases define: North, Northeast, South, Southeast, and West Central. For vertebrates, we use all terrestrial species known to breed in Brazil, not necessarily species endemic to it.

\section{Estimating the numbers of missing species}

The Joppa et al. (2010) model has two factors. First, the more taxonomists involved in describing species - the more species they will describe in a given interval, other things being equal. We define "taxonomists", simply, as those who describe new species. Taxonomic effort is a powerful predictor of the number of species described.

Second, taxonomists have likely increased the efficiency of their efforts since the mid-1700s when Linnaeus introduced the system of binomial nomenclature. By "taxonomic efficiency," Joppa et al. (2010) mean an increase in the number of species described per taxonomist, adjusted for the continually diminishing pool of as-yet unknown species. Were efficiency to have remained constant, the number of species described per taxonomist would decline continuously over time as the supply of un-described species dwindled. We shall show that for some taxa, there is an increase in the number of species per taxonomist, typically for a century or so.

For each five-year interval, we calculate the number of unique species discovered and the number of taxonomists working. We expect the number of species described in interval $S_{i}$ would depend on the number of taxonomists $\mathrm{T}_{\mathrm{i}}$ actively describing:

\section{$\mathrm{S}_{\mathrm{i}} \alpha \mathrm{T}_{\mathrm{i}}$}

Our model consists of two elements. The first is the remaining number of species to be described: $S_{R}$. It is the total number of species, $S_{T}$, - which is to be estimated - minus the cumulative number of species already described, $\Sigma \mathrm{S}_{\mathrm{i}}$ up to the given year, $\mathrm{t}$ :

$$
\mathrm{S}_{\mathrm{R}}=\mathrm{S}_{\mathrm{T}}-\sum \mathrm{S}_{\mathrm{i}} \text {, for } \mathrm{i}=1760 \text { to year } \mathrm{t} \text {. }
$$

We chose 1760 as the start date to avoid the undue influence of Linnaeus, who alone described many species in the previous decade (Linné 1753).

The second element is taxonomic efficiency, E. We assume that taxonomists have become more effective at finding and describing species now than in the past. For simplicity, we assume that this increase in efficiency increases linearly over time:

$$
\mathrm{E}_{\mathrm{i}}=\mathrm{a}+\mathrm{b} \times \text { year, or for convenience, } \mathrm{E}=\mathrm{a}+\mathrm{b} \mathrm{Y}_{\mathrm{i}}
$$

where $\mathrm{a}$ and $\mathrm{b}$ are also estimated parameters. Efficiency need not increase, whereupon $b$ would be zero. It follows that: 


$$
\frac{\mathrm{S}_{\mathrm{i}}}{\left[\mathrm{T}_{\mathrm{i}}\left(\mathrm{S}_{\mathrm{T}}-\sum \mathrm{S}_{\mathrm{i}}\right)\right]}=\left(\mathrm{a}+\mathrm{bY}_{\mathrm{i}}\right)
$$

This is an intrinsically non-linear statistical model, because there are four independent variables in the complete expression but only three parameters to be estimated. Nonetheless, it is easy to estimate the expression that minimizes the sum of the residual sums of squares between predicted and observed $S_{i}$. We guess a value of $S_{T}$, then calculate $\mathrm{a}$ and $\mathrm{b}$ from linear regression. Subsequent guesses of $\mathrm{S}_{\mathrm{T}}$ quickly converge on the minimum value.

The numbers of species described per period tends to be "spiky", indicating the undue influence of monographs that describe many species in the year they appear followed by intervals when taxonomists described relatively fewer species. To normalize the residuals, we took the logarithms of observed and predicted numbers of species and minimized the sums of squares of their differences. This logarithmic transformation creates large residuals when the numbers of species are very small, as they are in the mid-1700s. If at least 40 species had not been described by 1760 , we started in the first 5-year period where the cumulative number of known species was 40 or more.

\section{Range size and date of scientific description}

The vertebrate databases provide estimates of both range size and the date of species description. For the birds, a very few species with very small ranges were not included. We added estimates for these species from the most recent Birdlife website (http://www.birdlife.org/datazone/species/ index.html). Mitu mitu, now extinct in the wild, was given an arbitrary range of $1 \mathrm{~km}^{2}$. For reasons we shall presently explain, it is very likely that the data on range sizes and dates of description are incomplete for amphibians and mammals.

\section{Results and Discussion}

Figure 1 (right) shows the observed (blue) and predicted numbers (red) of selected families of species of flowering plants described per five-year interval and the numbers of taxonomists who described them for two regions of Brazil: Northern Brazil, which is mostly the Amazon, and South-eastern Brazil, which is mostly the Atlantic forest. There is a strong increase in the numbers of species described over time - which dooms any method to calculate total species numbers that assumes the rate will slow. There is also a marked increase in the numbers of taxonomists.

At the left are the numbers of species per taxonomist over time. The red lines show the best-fit predictions of the model. The numbers of species per taxonomist increase markedly initially for Northern Brazil and slightly for South-eastern Brazil. However, as the pool of unknown species decreases, the numbers of species per taxonomist drop markedly.
Figure 2 shows comparable plots for the mammals, amphibians, and birds found in Brazil. For mammals and birds, the data show strong declines in the number of species described per taxonomist.

Table 1 summarizes the data and the model predictions. For the plant families selected - which are not a complete inventory - South-eastern Brazil has by far the most endemic species $(>3,000)$. The model suggests that another $9 \%$ remain to be discovered. In contrast, the much larger northern region - mostly the Amazon - has far fewer species (740) and the model suggests that perhaps another $24 \%$ will be found. North-eastern Brazil has the highest predicted numbers of unknown species - $49 \%$ more - that reflect a more slowly declining number of species described by taxonomists over time.

As one might expect, the models predict very few bird and mammals remain unknown. Amphibians, in contrast, may produce another $15 \%$ of the present total of species - more than 100 new species.

For flowering plants, we do not have estimates of their range sizes. For the three vertebrate groups, we do. Figure 3 plots these against their dates of discovery. In the figure, each point represents a species. Mammals and birds are broadly similar. Before 1800 , taxonomists generally described species with geographical range sizes that exceed one million $\mathrm{km}^{2}$. These are not only widespread species, but they tend to be locally common (Pimm and Jenkins 2005, 2010). Since 1950 , however, the majority of newly described species have had ranges smaller than $100,000 \mathrm{~km}^{2}$. These tend to be locally rare (Pimm and Jenkins 2005, 2010).

Taxonomists described most of Brazil's mammals and birds before 1900. Amphibians are strikingly different. Amphibian species have much smaller ranges, on average, and the majority of species were described after 1950.

Figure 3 also shows the cumulative numbers of species with ranges $<20,000 \mathrm{~km}^{2}$. That small a range, combined with the levels of habitat destruction typical of Brazil outside the Amazon, would likely cause a species to be deemed "threatened" in the IUCN Redlist. Species with larger ranges but suffering other threats may also be so listed, of course. Moreover, analysis of bird species in the Atlantic forest and elsewhere suggests these range data greatly overestimate the likely range size when species' elevational ranges are used to refine the distribution maps (Harris \& Pimm 2008). We use this range size only as a simple benchmark.

For birds and mammals the overall numbers are relatively small, but more than $37 \%$ of all amphibians have ranges $<20,000 \mathrm{~km}^{2}$. What is also striking for amphibians, but also true for the smaller numbers of birds and mammals, is how recent are the discoveries of such small-ranged species. Half of all these small-ranged amphibians have been discovered since 1973, since 1972 for birds, and 1993 for mammals. 


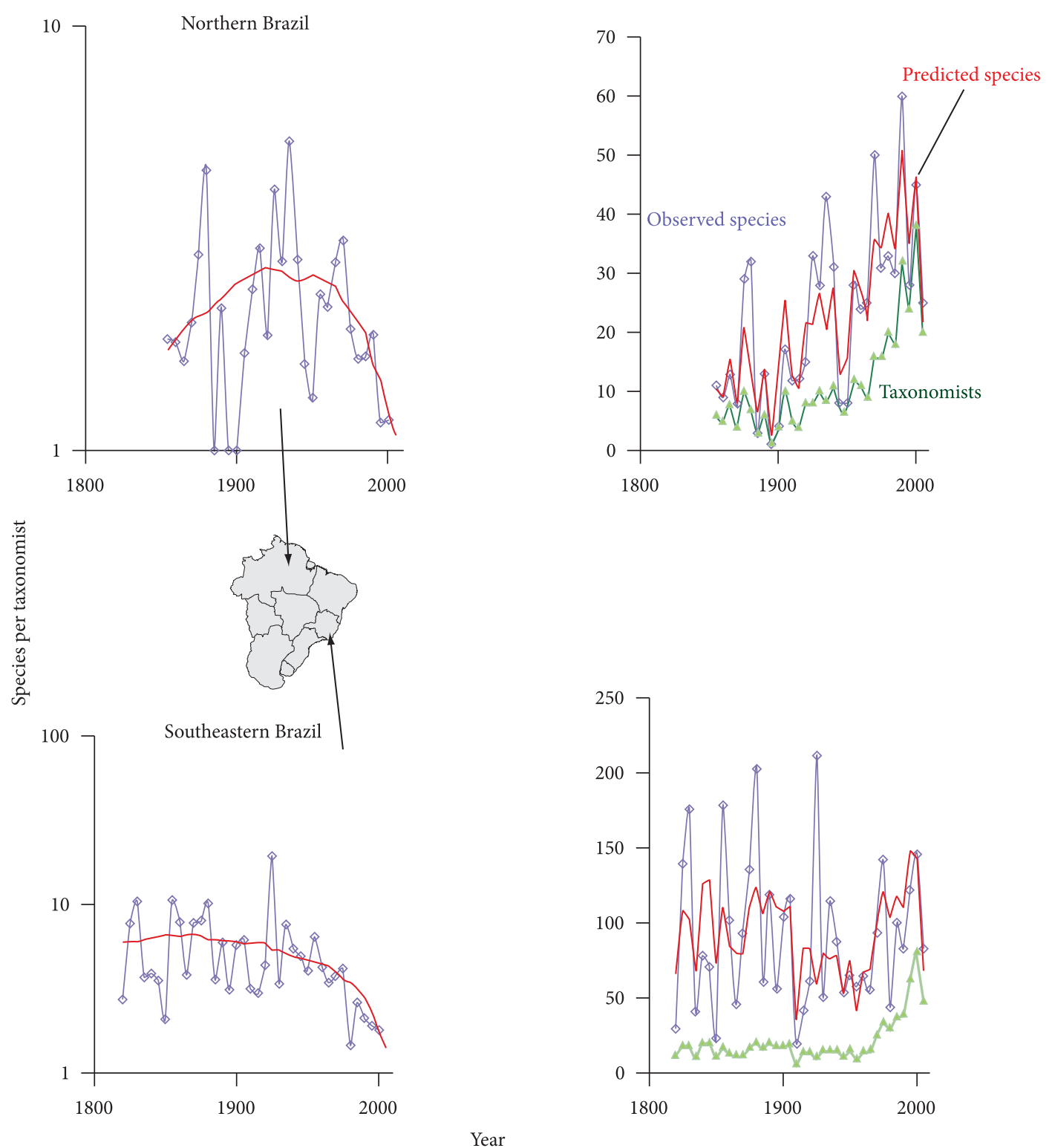

Figure 1. Left: The numbers of species of flowering plants described per five-year period divided by the number of taxonomists who described them (open symbols, blue lines) and the predicted values (red lines.) Right: the numbers of species of flowering plants described (open symbols, blue lines), their predicted values (red lines) and the number of taxonomists (green triangles).

\section{Implications for conservation}

Exercises in conservation priority setting usually assume that we know the species to be conserved as well as where they occur. [For the Atlantic forest, examples include Jenkins and Pimm (2006) and Jenkins et al. (2010).] So the issue of the species' lists being incomplete is an obvious one. It motivates our estimating how many species remain to be described. The numbers are small for birds and mammals. But 10 to $20 \%$ more amphibians and plants species may remain to be discovered. These percentages may still seem encouragingly low, given the complexities of studying a country as large as Brazil, with its extraordinary diversity, and with many of its regions large and still poorly explored.
What is more worrying is that these numbers of as-yet unknown species are broadly the same as the percentages of species that are presently considered to be threatened with extinction. That is, we likely know only half of the species that in reality are in danger of extinction. It is these species that matter when setting conservation priorities. Not only must we understand how many missing species there are, but we need to have some sense of where they are likely to be found. That second question is not one we address here. (Clearly, however, our knowledge of ranges of the rarest species is changing continuously as individual species are selected for detailed study (Alves et al. 2008, Vale et al. 2007).)

Does this mean that we do not know enough to conserve biodiversity and must wait until we have more complete 

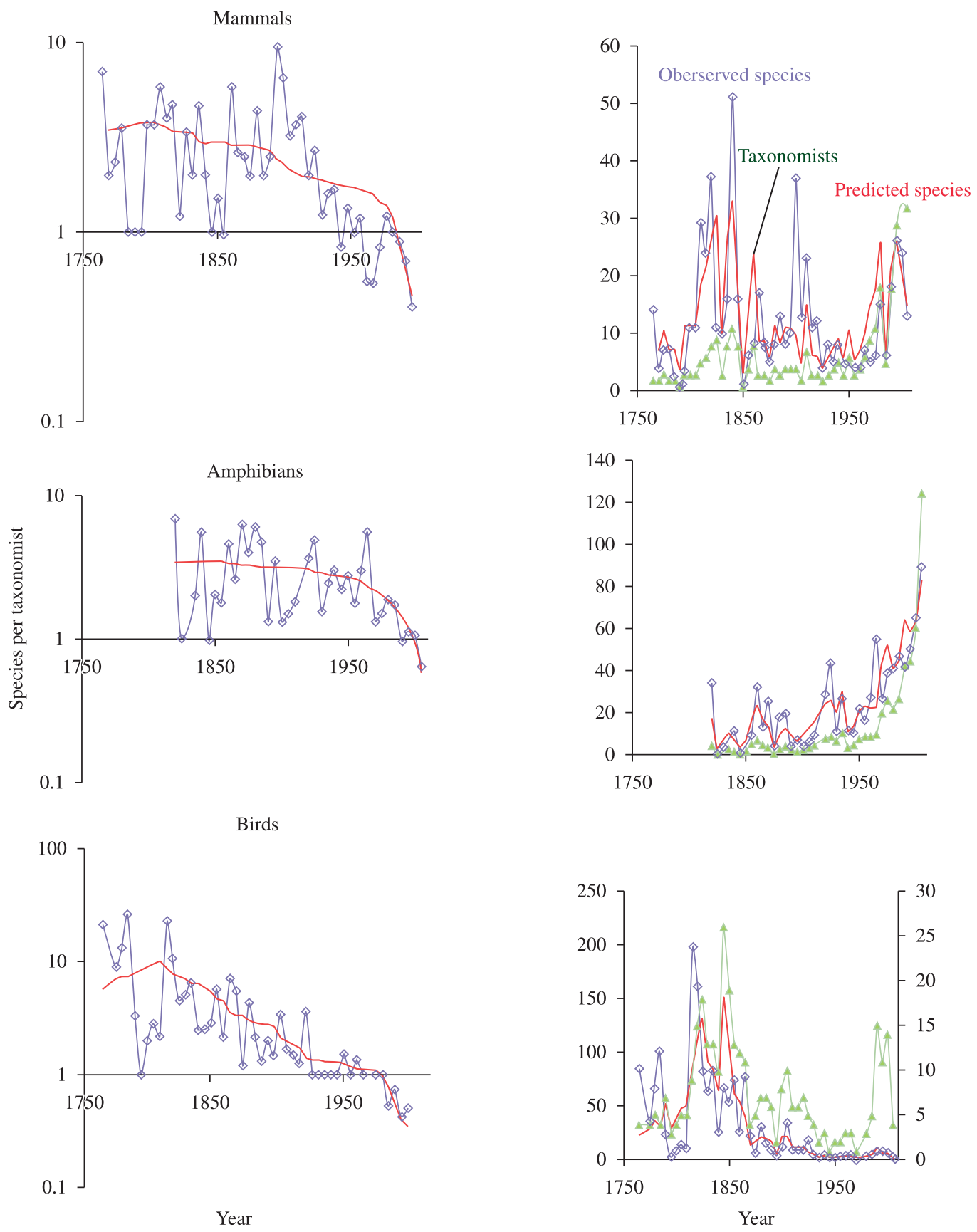

Figure 2. Left: The numbers of species described per five-year period divided by the number of taxonomists who described them (open symbols, blue lines) and the predicted values (red lines.) Right: the numbers of species described (open symbols, blue lines), their predicted values (red lines) and the number of taxonomists (green triangles). For birds, the number of taxonomists has a scale (right) different from that for the numbers of species.

taxonomic catalogues and maps of where the species occur? Clearly, the high rates of species losses do not permit such leisure: we must act quickly using the best available information.

That information can still be useful. All the plants and vertebrates we discuss have high levels of endemism in the Atlantic forest. Very little of it remains, what does remain is a conservation priority, and restoring the connections between isolated fragments of it is a most urgent requirement. Such actions are prudent even if the taxonomic catalogue is missing half its most threatened species. Moreover, it is likely that the missing species will be in the places that are already rich in endemic species, simply because that is the global pattern (Pimm and Jenkins 2005, 2010). While we expect that South-eastern Brazil will accumulate only another $9 \%$ more species, that is absolutely more species than we predict will be found in the Amazon. 
Table 1. Number of known and predicted species, and percentage of expected increase, of flowering plants (in different regions of Brazil) and vertebrates.

\begin{tabular}{lcccc}
\hline \multicolumn{1}{c}{ Region name } & Area $\left(\mathbf{k m}^{\mathbf{2}}\right)$ & Known species & Predicted & \%increase \\
\hline Flowering plants & & & & 689 \\
West Central & $1,608,042$ & 570 & 1518 & 49 \\
Northeastern Brazil & $1,544,347$ & 1016 & 3811 & 9 \\
Southeastern Brazil & 924,178 & 3485 & 914 & 24 \\
Northern Brazil & $3,832,654$ & 740 & 713 & 25 \\
Southern Brazil & 564,660 & 571 & & 15 \\
Vertebrates & & & 1017 & 6 \\
Amphibians & - & 887 & 658 & 1566 \\
Mammals & - & 620 & & 1 \\
Birds & - & 1554 & & \\
\hline
\end{tabular}
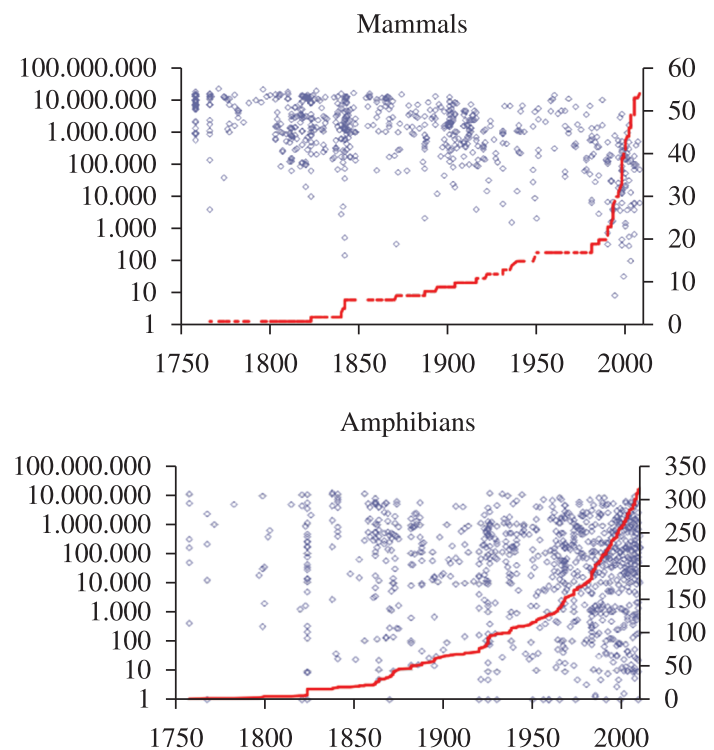

Birds

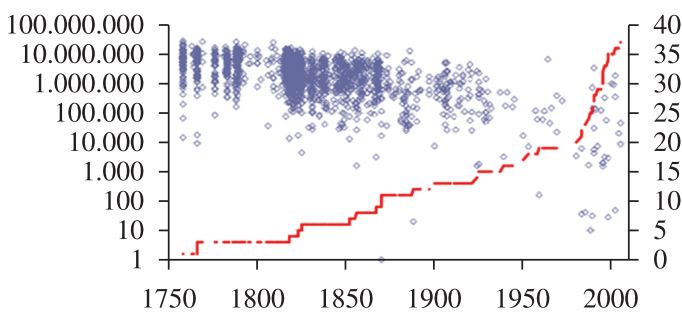

Figure 3. The geographical range size (in $\mathrm{km}^{2}$ ) of species versus the date of their description (solid blue points). The red lines show the cumulative number of species with ranges $<20,000 \mathrm{~km}^{2}$.

Nonetheless, the point of this exercise is to notice that our knowledge of species with small geographical ranges is particularly incomplete - and very recently acquired. Conservation professionals should follow the accumulation of new knowledge carefully. The central message of this paper is that conservation-relevant knowledge is still accumulating quickly, even for the best-known taxonomic groups.

\section{References}

Alves MAS, Pimm SL, Storni A, Raposo MA, Brooke M de L, Harris G et al., 2008. Mapping and exploring the distribution of a threatened bird, Grey-winged Cotinga. Oryx, 42:562-566.

Clayton, W.D., Harman, K.T. \& Williamson, H. 2009. GrassBase, The Online World Grass Flora. Available from: http://www. kew.org/data/grasses-db.html. [accessed May 2010]

Harris G \& Pimm SL, 2008. Range size and extinction risk in forest birds. Conservation Biology, 22:163-171.

IUCN, Conservation International, Arizona State University, Texas A\&M University, University of Rome, University of Virginia, et al., 2008a. An Analysis of Mammals on the 2008 IUCN Red List. [2009 January 25]. Available from: www. iucnredlist.org/mammals/.

IUCN, Conservation International \& NatureServe, 2008b. An analysis of amphibians on the 2008 IUCN Red List. [2009 February 10]. Available from: http://www.iucnredlist.org/ amphibians/.

Jenkins CN \& Pimm SL, 2006. Definindo Prioridades de Conservação em um Hotspot de Biodiversidade Global. In Rocha CFD, Bergallo HG, van Sluys M and Alves MAS (eds.). Biologia da Conservação: Essências. São Carlos: RiMa Editora.

Jenkins CN, Alves MAS \& Pimm SL, 2010. Avian conservation in a top-ranked biodiversity hotspot. Biological Conservation, 143:992-998.

Joppa LN, Roberts DL \& Pimm SL, 2010. How many species of flowering plants are there? Proceedings of the Royal Society $B$, doi: $10.1098 / \mathrm{rspb} .2010 .1004$.

Linné C, 1753 Species plantarum. Imprensis Laurentii Salvii, Stockholm, 2:970-971.

Pimm SL \& Jenkins C, 2005. Sustaining the variety of Life. Scientific American, (September):66-73.

Pimm SL \& Jenkins CN, 2010. Extinctions and the practice of preventing them. In Sodhi NS and Ehrlich PR (eds). Conservation Biology for All. Oxford: Oxford University Press. 
Ridgely RS, Allnutt TF, Brooks T, McNicol DK, Mehlman DW, Young BE, et al., 2007. Digital distribution maps of the birds of the Western Hemisphere. Version 3.0. Arlington, VA: NatureServe.

Solow A \& Smith W, 2005. On estimating the number of species from the discovery record. Proceedings of the Royal Society $B, 272: 285-287$.

Vale MM, Bell JB, Alves MAS \& Pimm SL, 2007. Abundance, distribution and conservation of Rio Branco Antbird Cercomacra carbonaria and Hoary-throated Spinetail Synallaxis kollari. Bird Conservation International, 17:245-257.
Wilson S \& Costello M, 2005. Predicting future discoveries of European marine species by using a non-homogeneous renewal process. Applied Statistics, 897-918.

Kew Plants People Possibilities, 2008. World Checklist of Selected Plant Families. The Board of Trustees of the Royal Botanic Gardens, Kew. Avaliable from: http://www.kew. org/wcsp/.
Received: June 2010

First Decision: June 2010

Accepted: June 2010 